\title{
Explicit Model Predictive Control of a Satellite with Magnetic Torquers
}

\author{
Thomas R. Krogstad Jan Tommy Gravdahl Petter Tøndel \\ Department of Engineering Cybernetics, \\ Norwegian University of Science and Technology \\ 7491 Trondheim, Norway \\ \{thomakro, tommy.gravdahl, petter.tondel\}@itk.ntnu.no
}

\begin{abstract}
In this paper we present the design of a linear constrained MPC controller for magnetic actuated small satellites. The controller may be derived by formulating a linear constrained MPC problem as a multi-parametric quadratic program (mpQP). The solution will be a piecewise affine (PWA) function, which may be evaluated at each sample to obtain the optimal control law. We apply this approach to the design of an explicit model predictive controller (eMPC) for the Norwegian student satellite nCube.
\end{abstract}

\section{INTRODUCTION}

The purpose of this paper is to derive an attitude controller for a small satellite actuated by means of magnetic coils. This is an area of research that has attracted much attention internationally. Noticeable contributions are [2] and [11], where nonlinear controllers have been proposed, a recent survey can be found in [16].

In order to handle the constraints on the available current and power, and limited computational power, we propose in this paper to solve the problem using an explicit model predictive control scheme. In [16] a MPC was proposed for magnetically controlled spacecraft, but to the best knowledge of the authors, the eMPC approach has not previously been applied to this problem. However, it has recently been used in [1] for a satellite actuated by means of thrusters and a reaction wheel. The eMPC approach retains MPC's ability to handle constraints, and in addition requires a small amount of online computational power. This property is obtained as the controller computation is solved offline, requiring only fixed-point arithmetics online, making it possible to realize the controller i embedded hardware.

For comparison in the simulations, we have implemented two nonlinear controllers [2] based on feedback from the angular velocity and attitude measurements, along with a measurement of the local magnetic field.

An outline of a stability proof using piecewise quadratic Lyapunov functions is proposed. This will however only show stability for each linearized model.

The results in this paper are based on the work in [3], where further details may be found.

\section{A. Explicit Model Predictive Control}

Model Predictive Control or MPC, involves solving a finite horizon optimal control problem at every time step.
The solution of this optimization problem is a series of control inputs for the whole horizon, giving an open loop controller. The control action computed for the first timestep is then applied to the plant, the horizon is shifted forward one time-step and the process is repeated, with the current state as initial values. In this manner MPC becomes a closed loop approach. Due to the shifting of the horizon this is also referred to as Receding Horizon Control or RHC. In this paper we will consider the linearized system

$$
\begin{aligned}
\mathbf{x}_{k+1} & =\mathbf{A} \mathbf{x}_{k}+\mathbf{B} \mathbf{u}_{k} \\
\mathbf{y}_{k} & =\mathbf{C} \mathbf{x}_{k},
\end{aligned}
$$

when designing the controller. Where $\mathbf{x}_{k} \in \mathbb{R}^{n}$ are the state variables, $\mathbf{u}_{k} \in \mathbb{R}^{m}$ are the input variables, $\mathbf{A} \in \mathbb{R}^{n \times n}$, $\mathbf{B} \in \mathbb{R}^{m \times m}$, and $(\mathbf{A}, \mathbf{B})$ is a stabilizable pair. In addition we may have hard constraints on both the states and inputs

$$
\begin{aligned}
& \mathbf{x}_{k} \in \mathbb{X} \\
& \mathbf{u}_{k} \in \mathbb{U}
\end{aligned}
$$

where $\mathbb{X}$ is a convex closed subset of $\mathbb{R}^{n}$ and $\mathbb{U}$ is a convex compact subset of $\mathbb{R}^{m}$, both containing the origin in the interior. A terminal constraint may also be imposed for stability reasons,

$$
\mathbf{x}_{k+N} \in X_{f} \subset \mathbb{X}
$$

where $\mathrm{N}$ is the prediction horizon.

If we now consider the regulator problem, that is, the problem of driving the state vector to the origin, the traditional MPC solves the following convex optimization problem for the current $\mathbf{x}_{k}$

$$
\begin{array}{ll}
\min _{\mathbf{U}, \mathbf{s}}[ & {\left[J(\mathbf{U}, \mathbf{x}(t))+\rho\|\mathbf{s}\|_{\mathcal{L}_{2}}^{2}\right]} \\
\text { s.t. } & \mathbf{y}_{\min }-\mathbf{s} \leq \mathbf{y}_{t+k \mid t} \leq \mathbf{y}_{\max }+\mathbf{s}, k=1, \ldots, N \\
& \mathbf{u}_{\min } \leq \mathbf{u}_{t+k} \leq \mathbf{u}_{\max }, k=0, \ldots, M-1 \\
& \mathbf{u}_{t+k}=\mathbf{K} \mathbf{x}_{t+k \mid t}, M \leq k \leq N-1 \\
& \mathbf{x}_{t \mid t}=\mathbf{x}(t) \\
& \mathbf{x}_{t+k+1 \mid t}=\mathbf{A} \mathbf{x}_{t+k \mid t}+\mathbf{B} \mathbf{u}_{t+k}, k \geq 0 \\
& \mathbf{y}_{t+k \mid t}=\mathbf{C x}_{t+k \mid t}, k \geq 0
\end{array}
$$


where $J(\mathbf{U}, \mathbf{x}(t))$ is the quadratic cost function

$$
\begin{aligned}
J(\mathbf{U}, \mathbf{x}(t))= & \mathbf{x}_{t+N \mid t}^{T} \mathbf{P} \mathbf{x}_{t+N \mid t} \\
& +\sum_{k=0}^{N-1} \mathbf{x}_{t+k \mid t}^{T} \mathbf{Q} \mathbf{x}_{t+k \mid t}+\mathbf{u}_{t+k}^{T} \mathbf{R} \mathbf{u}_{t+k},
\end{aligned}
$$

$\|\mathbf{s}\|_{\mathcal{L}_{2}}$ is the $\mathcal{L}_{2}$-norm of the slack variables $\mathbf{s}, \rho$ is the penalty weight of the slack variables, $\mathbf{U} \triangleq\left[\mathbf{u}(k)^{T}, \mathbf{u}(k+\right.$ $\left.1)^{T}, \ldots, \mathbf{u}(k+N-1)^{T}\right]^{T}$ is the vector of inputs at each sample time, $\mathbf{s} \triangleq\left[\mathbf{s}^{T}(k), \ldots, \mathbf{s}^{T}(k+N-1)\right]^{T}$ is the vector of slack variables, $\mathbf{K}$ is the control gain matrix when the input is unconstrained, $\mathbf{x}_{t+k \mid t}$ is the prediction of $\mathbf{x}_{t+k}$ at time $t$, and $N$ and $M$ are the output and input constraint horizons respectively. $\mathbf{P} \in \mathbb{R}^{n \times n}, \mathbf{P}=\mathbf{P}^{T} \geq 0, \mathbf{R} \in$ $\mathbb{R}^{m \times m}, \mathbf{R}=\mathbf{R}^{T} \geq 0, \mathbf{Q} \in \mathbb{R}^{n \times n}, \mathbf{Q}=\mathbf{Q}^{T} \geq 0$. The final-state weight matrix $\mathbf{P}$ is typically computed using the algebraic Riccati equation. The solution to (5) is now given as: $\mathbf{U}^{*}=\left[\mathbf{u}^{*}(k)^{T}, \mathbf{u}^{*}(k+1)^{T}, \ldots, \mathbf{u}^{*}(k+N-1)^{T}\right]^{T}, \mathbf{s}^{*}=$ $\left[\mathbf{s}^{*}(k)^{T}, \ldots, \mathbf{s}^{*}(k+N-1)^{T}\right]^{T}$

In order to compute the explicit MPC controller, we need to formulate the linear MPC problem as an mpQP problem. The details of the derivation are given in [5], [6]. By some algebraic manipulation the problem may be reformulated as

$$
\begin{aligned}
& V_{z}(\mathbf{x})=\min _{z} \frac{1}{2} \mathbf{z}^{T} \mathbf{H z} \\
& \text { s.t } \quad \mathbf{G z} \leq \mathbf{W}+\mathbf{S x}(t),
\end{aligned}
$$

where $\mathbf{z} \triangleq \mathbf{U}+\mathbf{H}^{-1} \mathbf{F}^{T} \mathbf{x}(t), \mathbf{x}(t)$ is the current state, which can be treated as a vector of parameters to the optimization problem. Note that $\mathbf{H} \succ 0$ since $\mathbf{R} \succ 0$. The number of inequalities is denoted by $\mathrm{q}$ and the number of free variables is $n_{z}=m \cdot N$. Then $\mathbf{z} \in \mathbb{R}^{n_{z}}, \mathbf{H} \in \mathbb{R}^{n_{z} \times n_{z}}, \mathbf{G} \in \mathbb{R}^{q \times n_{z}}$, $\mathbf{W} \in \mathbb{R}^{q \times 1}, \mathbf{S} \in \mathbb{R}^{q \times n}, \mathbf{F} \in \mathbb{R}^{n \times q}$. The optimization problem (7)-(8) in now considered to be an mpQP, meaning that we seek a solution on explicit form, as a function of the parameter $\mathbf{x}(t)$.

The task is now to find the explicit solution of this mpQP problem, $\mathbf{z}^{*}=\mathbf{z}^{*}(\mathbf{x}(t))$, so that we may use the definition of $\mathbf{z}$ to find the explicit controller $\mathbf{U}^{*}$ as a function of the state vector. As shown in [6], this can be solved by applying the Karush-Kuhn-Tucker (KKT) conditions. Where the KKT-conditions are necessary and sufficient for an optimal solution for a convex quadratic problem [10].

The solution will then be a continuous PWA function, defined over a polyhedral partition of the parameter space. Which may be evaluated at each sample to obtain the control input

$$
\mathbf{u}_{k}=\mathbf{K}_{i} \mathbf{x}_{k}+\mathbf{k}_{i}, \forall \mathbf{x}_{k} \in \mathcal{X}_{i}
$$

where $\mathbf{K}_{i}$ is the gain-matrix for region $i, \mathbf{k}_{i}$ is a constant vector, $\mathbf{x}_{k}$ the current state and $\mathcal{X}_{i}$ is the $\mathrm{i}$ 'th region.

\section{B. Electromagnetic Actuators}

Electromagnetic actuators are often chosen due to the independence of a limited fuel source, depending instead on power from solar arrays and batteries and thereby prolonging the lifespan of the satellite. Electromagnetic actuators, often referred to as magnetic torquers, are based on two basic configurations. One is the coil based, where current is sent through a current loop which generates the magnetic moment proportional to the area of the coil and the number of windings. The other type is the magnetic rod, where wire is winded around a rod made of a high permeability material. Both variations interact with the local geomagnetic field, generating a torque vector in the direction perpendicular to the magnetic moment vector and the local field direction.

\section{MODELLING}

In this section, the model of a satellite actuated by means of electromagnetic torquers will be derived. The notation is based on [7] and [8].

\section{A. Kinematics}

We will describe the attitude kinematics in the form of Euler parameters, which may be defined from the angle-axis parameters $\theta$ and $\mathbf{k}$

$$
\eta=\cos \frac{\theta}{2}, \quad \epsilon=\mathbf{k} \sin \frac{\theta}{2},
$$

which gives the corresponding rotation matrix

$$
\mathbf{R}(\eta, \boldsymbol{\epsilon})=\mathbf{1}+2 \eta \boldsymbol{\epsilon}^{\times}+2 \boldsymbol{\epsilon}^{\times} \boldsymbol{\epsilon}^{\times}
$$

where ${ }^{\times}$denotes the vector cross product operator, and $\epsilon^{\times}$ is skew-symmetric. The choice of Euler parameters is motivated by their properties as a nonsingular representation.

From the properties of the rotation matrix, it can be shown that

$$
\dot{\mathbf{R}}_{o}^{b}=\left(\boldsymbol{\omega}_{b o}^{b}\right)^{\times} \mathbf{R}_{o}^{b}=-\left(\boldsymbol{\omega}_{o b}^{b}\right)^{\times} \mathbf{R}_{o}^{b},
$$

where $\boldsymbol{\omega}_{b o}^{b}$ is the angular velocity of the body frame $\mathcal{F}_{b}$ with respect to the orbit frame $\mathcal{F}_{o}$, and $\mathbf{R}_{o}^{b}$ is the rotation matrix between frames. $\mathcal{F}_{o}$ has its origin in the satellites center of mass, its z-axis always pointing towards Earth (nadir direction), its $y$-axis is chosen in the direction of the negative orbit normal and finally the $\mathrm{x}$-axis is chosen in order to complete a right-handed system.

Using (11) and (12) the kinematic differential equations

$$
\begin{aligned}
& \dot{\eta}=-\frac{1}{2} \boldsymbol{\epsilon}^{T} \boldsymbol{\omega}_{o b}^{b} \\
& \dot{\boldsymbol{\epsilon}}=\frac{1}{2}\left[\eta \mathbf{I}+\boldsymbol{\epsilon}^{\times}\right] \boldsymbol{\omega}_{o b}^{b},
\end{aligned}
$$

can be derived.

\section{B. Dynamics}

Assuming that the satellite is a rigid body, with the body coordinate frame coinciding with the principal axes, we may write the attitude dynamics as [9]

$$
\mathbf{I} \dot{\boldsymbol{\omega}}_{i b}^{b}+\left(\boldsymbol{\omega}_{i b}^{b}\right)^{\times} \mathbf{I} \boldsymbol{\omega}_{i b}^{b}=\boldsymbol{\tau}_{e}^{b},
$$

where $\mathbf{I}=\operatorname{diag}\left(i_{x x}, i_{y y}, i_{z z}\right)$ is the inertia matrix, $\boldsymbol{\omega}_{i b}^{b}$ is the body frame's angular velocity relative to the inertial frame $\mathcal{F}_{i}$, and $\tau_{e}^{b}$ is the external torque given in $\mathcal{F}_{b}$. 
Further, we assume that the only external torques of importance are the gravity gradient $\tau_{g}^{b}$ and the torque $\tau_{m}^{b}$ supplied by the actuators:

$$
\begin{aligned}
\boldsymbol{\tau}_{g}^{b} & =3 \omega_{o}^{2}\left(\mathbf{z}_{o 3}^{b}\right)^{\times} \mathbf{I}\left(\mathbf{z}_{o 3}^{b}\right) \\
\boldsymbol{\tau}_{m}^{b} & =\left(\mathbf{m}^{b}\right)^{\times} \mathbf{B}^{b}(t),
\end{aligned}
$$

where $\omega_{o}$ is the satellites angular velocity about the earth, assuming a circular orbit, $\mathbf{z}_{o 3}^{b}$ is the earth pointing vector, $\mathbf{m}^{b}$ is the magnetic moment exerted by the actuators and $\mathbf{B}^{b}(t)$ is the geomagnetic field.

Since we are concerned with the satellite's orientation relative to $\mathcal{F}_{o}$, we would also like the model to represent the angular velocity of $\mathcal{F}_{b}$ relative to this frame. Using the assumption of a circular orbit, we define the relationship between the angular velocities as

$$
\boldsymbol{\omega}_{i b}^{b}=\boldsymbol{\omega}_{o b}^{b}+\mathbf{R}_{o}^{b} \boldsymbol{\omega}_{i o}^{o} \quad \text { and } \quad \dot{\boldsymbol{\omega}}_{i b}^{b}=\dot{\boldsymbol{\omega}}_{o b}^{b}+\dot{\mathbf{R}}_{o}^{b} \boldsymbol{\omega}_{i o}^{o},
$$

where $\boldsymbol{\omega}_{i o}^{o}=\left[\begin{array}{lll}0 & -\omega_{o} & 0\end{array}\right]^{T}$.

Inserting (15a), (15b) and (16) into (14), we may express the complete nonlinear dynamics as

$$
\begin{aligned}
\dot{\boldsymbol{\omega}}_{o b}^{b} & =-\mathbf{I}^{-1}\left(\boldsymbol{\omega}_{o b}^{b}+\mathbf{R}_{b}^{o} \boldsymbol{\omega}_{\boldsymbol{i o}}^{\mathbf{o}}\right)^{\times} \mathbf{I}\left(\boldsymbol{\omega}_{o b}^{b}+\mathbf{R}_{b}^{o} \boldsymbol{\omega}_{\boldsymbol{i o}}^{\mathbf{o}}\right) \\
& +3 \omega_{o}^{2} \mathbf{I}^{-1}\left(\mathbf{z}_{o 3}^{b}\right)^{\times} \mathbf{I}\left(\mathbf{z}_{o 3}^{b}\right)+\left(\omega_{o b}^{b}\right)^{\times} \mathbf{R}_{o}^{b} \boldsymbol{\omega}_{i o}^{o} \\
& +\mathbf{I}^{-1}\left(\mathbf{m}^{b}\right)^{\times} \mathbf{B}^{b}(t) .
\end{aligned}
$$

\section{Linearizing}

In order to derive the eMPC controller we linearize the nonlinear model. Define a state vector $\mathbf{x} \triangleq\left[\left(\boldsymbol{\omega}_{o b}^{b}\right)^{T}, \eta, \boldsymbol{\epsilon}^{T}\right]^{T}$ and an input vector $\mathbf{u} \triangleq \mathbf{m}^{b}$. The complete nonlinear model can now be written as $\dot{\mathbf{x}}=\mathbf{f}(t, \mathbf{x}, \mathbf{u})$. Using Taylor expansion we linearize the model about the set-point $\mathbf{x}_{p}=[0,0,0,1,0,0,0]^{T}$ and

$\mathbf{u}_{p}=[0,0,0]^{T}$, and obtain the linear model:

$$
\Delta \dot{\mathbf{x}}=\mathbf{A}_{c} \Delta \mathbf{x}+\mathbf{B}_{c} \Delta \mathbf{u}
$$

where $\mathbf{A}_{c}$ and $\mathbf{B}_{c}$ are given by

$$
\left.\mathbf{A}_{c} \triangleq \frac{\partial \mathbf{f}}{\partial \mathbf{x}}\right|_{\mathbf{x}_{p}} \quad \text { and }\left.\quad \mathbf{B}_{c} \triangleq \frac{\partial \mathbf{f}}{\partial \mathbf{u}}\right|_{\mathbf{u}_{p}},
$$

where

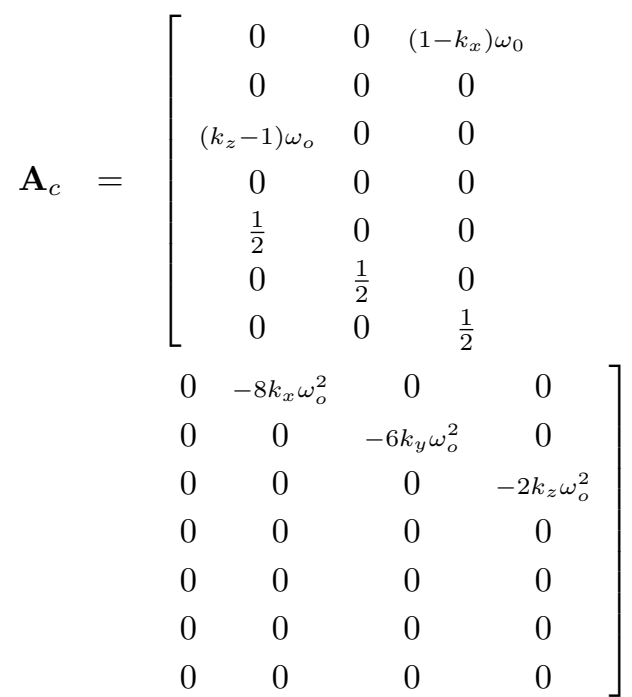

and

$$
\mathbf{B}_{c}=\left[\begin{array}{ccc}
0 & 0 & 0 \\
0 & 0 & 0 \\
0 & 0 & 0 \\
0 & 0 & 0 \\
0 & \frac{B_{z}^{b}}{i_{x x}} & -\frac{B_{y}^{b}}{i_{x x}} \\
-\frac{B_{z}^{b}}{i_{y y}} & 0 & \frac{B_{x}^{b}}{i_{y y}} \\
\frac{B_{y}^{b}}{i_{z z}} & -\frac{B_{x}^{b}}{i_{z z}} & 0
\end{array}\right],
$$

where $k_{x}=\frac{i_{y y}-i_{z z}}{i_{x x}}, k_{y}=\frac{i_{x x}-i_{z z}}{i_{y y}}$, and $k_{z}=\frac{i_{y y}-i_{x x}}{i_{z z}}$.

\section{CONTROL DESIGN}

\section{A. Explicit MPC controller}

In this section we will derive the eMPC controller for the electromagnetic actuated satellite. Upon inspection of the linear model, we see that the state $\eta$ is uncontrollable. However using the quaternion redundancy $\eta^{2}+\boldsymbol{\epsilon}^{T} \boldsymbol{\epsilon}=1$, we see that we may control $\eta$ through $\epsilon$. We may therefore exclude $\eta$ from the rest of the analysis and design.

Another issue is the time varying input matrix $\mathbf{B}_{c}$. This matrix is dependent on the local magnetic field in $\mathcal{F}_{b}$. From the model of the magnetic field, we know that this varies periodically about 0 when measured in $\mathcal{F}_{o}$. Assuming small attitude deviations from the set-point, $\mathcal{F}_{b}$ coincides with $\mathcal{F}_{o}$, and $\mathbf{B}^{b}$ may be set equal to $\mathbf{B}^{o}$. A problem that arises is when the field changes sign, resulting in a positive feedback loop. One solution is to estimate the absolute value of the field strength along each axis, make 8 input matrices for each combination of signs and create one controller for each resulting model. Since the sign of the local field is measured, we may switch between the appropriate controllers.

Next, we define a proper scaling of the model. This is important due to numerical sensitivity in the mpQP algorithm, which may occur when there are large differences in the order of magnitude between the matrix elements. To 
obtain the scaled model we first define the scaled variables and inputs $\overline{\mathbf{x}} \triangleq \mathbf{N}_{x}^{-1} \mathbf{x}$ and $\overline{\mathbf{u}} \triangleq \mathbf{N}_{u}^{-1} \mathbf{u}$, where the scaling matrices are defined as

$$
\begin{aligned}
& \mathbf{N}_{x}=\operatorname{diag}\left(\left[\begin{array}{llllll}
1 & 1 & 1 & 10^{-3} & 10^{-3} & 10^{-3}
\end{array}\right]\right) \\
& \mathbf{N}_{u}=\operatorname{diag}\left(\left[\begin{array}{llll}
0.1 & 0.1 & 0.1
\end{array}\right]\right) .
\end{aligned}
$$

A scaled model may then be written

$$
\begin{aligned}
\dot{\overline{\mathbf{x}}} & =\mathbf{N}_{x}^{-1} \mathbf{A} \mathbf{N}_{x} \overline{\mathbf{x}}+\mathbf{N}_{x}^{-1} \mathbf{B} \mathbf{N}_{u} \overline{\mathbf{u}} \\
& =\overline{\mathbf{A}} \overline{\mathbf{x}}+\overline{\mathbf{B}} \overline{\mathbf{u}} .
\end{aligned}
$$

Using the scaled model, we discretize the system with a time-step $T_{s}$ of 0.5 seconds, using the first order hold method.

TABLE I

ALGORITHM PARAMETERS

\begin{tabular}{c||c}
\hline Parameter & Value \\
\hline \hline $\mathbf{Q}$ & $\operatorname{diag}\{10,100,100,10,10,10\}$ \\
$\mathbf{R}$ & $\operatorname{diag}\{100000,100000,100000\}$ \\
$N$ (Horizon) & 1 \\
$\boldsymbol{\rho}$ & $-[10,10,10,1,1,1]^{T} \leq$ \\
Parameter space & $\mathbf{x} \leq[10,10,10,1,1,1]^{T}$ \\
Actuator constraints & $-1 \leq u_{i} \leq 1 \forall i \in\{1,2,3\}$
\end{tabular}

Using the parameters in Table I, we employ the mpQP algorithm. The solution is a polyhedral partitioning of the parameter space $\mathbb{X}$, into 21 regions, where for each region the optimal linear state-feedback control law is given by (9) The solution is thus 8 PWA controllers which are scheduled according to the measured sign of the magnetic field.

\section{B. Nonlinear controllers}

To asses the performance of the eMPC controller, we have implemented two nonlinear controllers (23) and (24), which based on feedback from the angular velocity and attitude [2]:

$$
\begin{gathered}
\mathbf{m}^{b}=h \boldsymbol{\omega}_{o b}^{b} \times \mathbf{B}^{b} \\
\mathbf{m}^{b}=h \boldsymbol{\omega}_{o b}^{b} \times \mathbf{B}^{b}-\alpha \boldsymbol{\epsilon} \times \mathbf{B}^{b}
\end{gathered}
$$

where $h>0$ and $\alpha>0$ are constants.

\section{Stability}

Stability of the linearized system with eMPC control can be derived using a piecewise quadratic Lyapunov function, as suggested by [14], by defining the closed-loop system as a PWA system:

$$
\mathbf{x}_{k+1}=\mathbf{A}_{i} \mathbf{x}_{k}+\mathbf{a}_{i}, \forall \mathbf{x}_{k} \in \mathcal{X}_{i}
$$

where $\mathbf{A}_{i} \in \mathbb{R}^{n \times n}, \mathbf{a}_{i} \in \mathbb{R}^{n}$ and the state belongs to the set of states $\mathbf{X} \subset \mathbb{R}^{n}$. The set of cells $\left\{\mathcal{X}_{i}\right\}_{i=1}^{s}$ represent a polyhedral partition of $\mathbf{X}$, i.e. each set $\mathcal{X}_{i}$ is a (not necessarily closed) convex polyhedron such that the origin belongs to $\mathbf{X}$.
In [14] $\mathcal{S}$ - PWQ stable with relaxations, is presented as the least conservative criterion:

$$
\begin{gathered}
\mathbf{P}_{i}-\overline{\mathbf{E}}_{i}^{T} \overline{\mathbf{U}}_{i} \overline{\mathbf{E}}_{i}>0, \forall i \in \mathcal{I} \\
\overline{\mathbf{A}}_{j}^{T} \mathbf{P}_{i} \overline{\mathbf{A}}_{j}-\gamma \mathbf{P}_{j}+\overline{\mathbf{E}}_{i}^{T} \overline{\mathbf{Z}}_{i j} \overline{\mathbf{E}}_{i}<0, \forall(i, j) \in \mathcal{S},
\end{gathered}
$$

where $\mathcal{I}$ is the set of indices denoting the regions of the state-space, and $\mathcal{S}$ denotes the set of ordered pairs $(i, j)$ of possible transitions between regions. If we can find a feasible solution $\mathbf{P}_{i}=\mathbf{P}_{i}^{T}, \overline{\mathbf{U}}_{i}$ and $\overline{\mathbf{Z}}_{i j}$ for this LMI, the origin is exponentially stable on $\mathbb{X}_{0}$ with a degree $\gamma$. We may now refer to the system as $\mathcal{S}-P W Q$ stable with relaxations.

The stability of the satellite system is currently being investigated.

\section{Simulations}

In this section we present simulations of the different controllers. The Norwegian student satellite is used as a case. Further information on this project can be found in [12] and [13]. The simulation parameters are summarized in Table II.

\begin{tabular}{|c|c|}
\hline Parameter & Value \\
\hline Inertia matrix & $\begin{array}{c}\operatorname{diag}\{0.1043,0.1020,0.0031\} \\
{\left[\mathrm{kgm}^{2}\right]}\end{array}$ \\
\hline Maximal magnetic moment & $0.1\left[A m^{2}\right]$ \\
\hline Desired Euler angles & {$\left[\begin{array}{lll}0 & 0 & 0\end{array}\right]^{T}[\mathrm{deg}]$} \\
\hline Desired angular velocity & {$\left[\begin{array}{lll}0 & 0 & 0\end{array}\right]^{T}[\mathrm{rad} / \mathrm{s}]$} \\
\hline Pointing accuracy required & $\pm 10^{\circ}$ on roll and pitch \\
\hline Orbit angular velocity $-\omega_{o}$ & $1.083 \times 10^{-3}[\mathrm{rad} / \mathrm{s}]$ \\
\hline Orbit period & $5801.6[\mathrm{sec}]$ \\
\hline Initial attitude & $\Phi=[20,40,60]^{T}[\mathrm{Deg}]$ \\
\hline Initial angular velocity & $\boldsymbol{\omega}_{o b}^{b}=[5,-3,3]^{T} \times 10^{-3}[\mathrm{rad} / \mathrm{s}]$ \\
\hline
\end{tabular}

In the remainder the controller parameters for the nonlinear controllers are

$$
\begin{aligned}
& h=2.25 \times 10^{5} \\
& \alpha=450 .
\end{aligned}
$$

The controllers were tuned for best possible performance. The eMPC controller is derived using the parameters of Table I.

TABLE II

SIMULATION PARAMETERS

The model is simulated with the presence of noise on the inputs to simulate disturbance torques and on the measurements of the magnetic field. The magnetic field values is generated using an orbit propagator and the IGRF 2000 model of the Earth's magnetic field [15].

As can be seen from fig. 1 and 2, both the eMPC controller and the nonlinear controller 2, manages to steer the state to the required accuracy. However, the nonlinear controller 1 points the satellite in the negative nadir direction. 

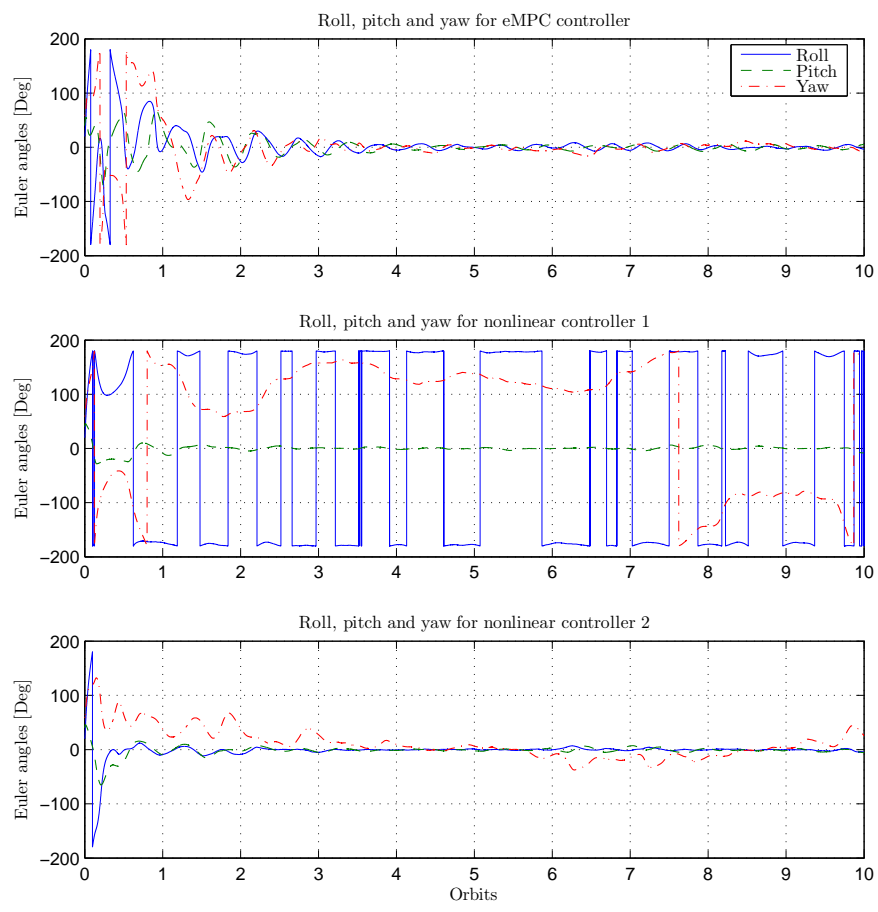

Fig. 1. The Euler angles

Fig. 4 and 3 shows that the energy consumption is clearly decreased in the case of the eMPC controller, and shows a lower peak in the power drawn from the power supply. An attempt was made to tune the nonlinear controller 2, through the variables $h$ and $\alpha$, in order to minimize the energy consumption while keeping the desired accuracy. The consumption was decreased to about $8 \mathrm{~J}$ for 10 orbits, thus still higher than with eMPC control. On the other hand, the control law (24) results in the fastest convergence of the three controllers.

\section{DISCUSSION}

It is clear that although the proposed controller achieves the required accuracy in roll and pitch, the nonlinear controller with attitude feedback does outperform it, at the cost of higher energy consumption. In particular the transient response is faster, and a greater accuracy is maintained at steady-state.

Nonlinear model predictive control (NMPC) would probably outperform the proposed controller, but the computational requirements for NMPC makes this an infeasible solution for satellite control.

\section{CONCLUSIONS}

We have in this paper presented the design of an explicit model predictive controller and compared it to other possible control schemes. The results show a decrease in total energy consumption while still maintaining the desired accuracy. Hence it has been shown to be a highly attractive solution for satellite control, where energy consumption is of the greatest importance.
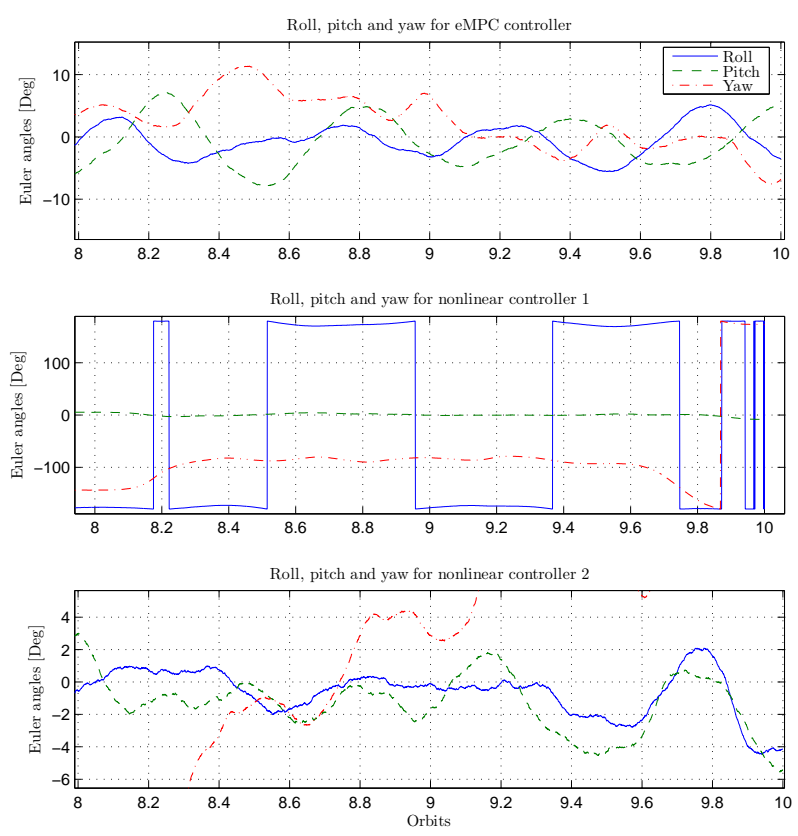

Fig. 2. The Euler angles, zoomed in on the 2 last orbits

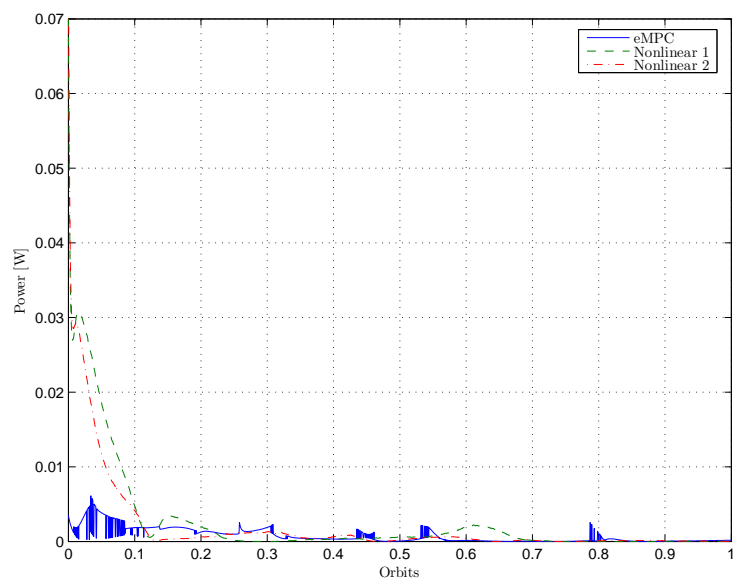

Fig. 3. Power usage in coils, zoomed in on the first orbit

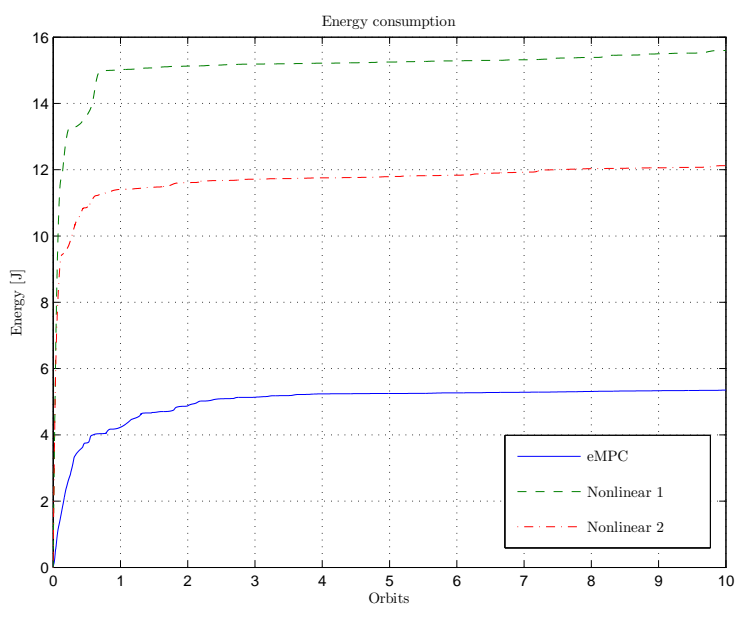

Fig. 4. Energy consumption 


\section{REFERENCES}

[1] Hegrenæs, Ø., "Attitude Control by means of Explicit Model Predictive Control, via Multi-parametric Quadratic Programming", $M S c$. thesis, Norwegian University of Science and Technology, (2004).

[2] Wiśniewski, R. and Blanke, M., "Fully magnetic attitude control for spacecraft subject to gravity gradient", Automatica, Vol. 35, pp. 12011214, (1999).

[3] Krogstad, T. R., "Attitude Control and Stability Analysis of Satellites in Earth and Moon Orbits", Msc. Project work, Norwegian University of Science and Technology, (2004)

[4] Tøndel, P., T. A. Johansen and A. Bemporad, "An algorithm for multiparametric quadratic programming and explicit MPC solutions", Automatica, Vol. 39, pp. 489-497, (2003).

[5] Tøndel, P., "Constrained Optimal Control via Multiparametric Quadratic Programming", Ph.D. Thesis, Norwegian University of Science and Technology, (2003).

[6] Bemporad, A., M. Morari, V. Dua and E. N. Pistikopoulos, "The explicit linear quadratic regulator for constrained systems", Automatica, Vol. 38, pp. 3-20, (2002).

[7] Hughes, P. C., "Spacecraft attitude dynamics", John Wiley \& Sons, (1986).

[8] Egeland, O. and J. T. Gravdahl, "Modeling and Simulation for Automatic Control", Marine Cybernetics, Trondheim, Norway, (2002).

[9] Fossen, T.I., "Matematiske modeller for styring av fly og satelitter", Technical report, Norwegian University of Science and Technology, (1998).

[10] Nocedal, J. and Wright, S.J., "Numerical Opimization", SpringerVerlag, (1999).

[11] Lovera, M. and Astolfi, A., "Spacecraft Attitude Control Using Magnetic Actuators", Automatica, Vol. 40, pp. 1405-1414, (2004).

[12] Riise, Å.-R., Samuelsen, B., Sokolova, N., Cederblad, H., Fasseland, J., Nordin, C., Otterstad, J., Fauske, K., Eriksen, O., Indergaard, F., Svartveit, K., Furebotten, P., Sæther, E., and Eide, E., "Ncube: The First Norwegian Student Satellite", In Proceedings of The 17th AIAA/USU Conference on Small Satellites, Logan, Utah, August, (2003)

[13] Gravdahl, J.T, E. Eide, A.Skavhaug, K. Svartveit, K. M. Fauske and F. M. Indergaard, "Three axis Attitude Determination and Control System for a picosatellite: Design and implementation", Proceedings of the 54th International Astronautical Congress, Bremen, Germany, 2003

[14] Ferrari-Trecate, G., Cuzzola, F. A., Mignone, D. and Morari, M., "Analysis of disrete-time piecewise affine and hybrid systems", Automatica, Vol. 38, pp. 2139-2146, (2002)

[15] Vallado,D. A., "Fundamentals of Astrodynamics and Applications", Kluwer Academic Publishers, (2001)

[16] Silani,E., M. Lovera "Magnetic spacecraft attitude control: a survey and some new results", Control Engineering Practice, Vol. 13, pp. 357-371, (2005) 